\begin{tabular}{|c|c|}
\hline Title & $\begin{array}{l}\text { Precise Measurement of IMD Behavior in 5-GHz HTS Resonators and Evaluation of Nonlinear Microwave } \\
\text { Characteristics }\end{array}$ \\
\hline Author(s) & $\begin{array}{l}\text { Futatsumori, Shunichi; Furuno, Masato; Hikage, Takashi; Nojima, Toshio; A kasegawa, A kihiko; Nakanishi, Teru; } \\
\text { Y amanaka, Kazunori }\end{array}$ \\
\hline Citation & $\begin{array}{l}\text { IEEE Transactions on A pplied Superconductivity, 19(3), 3595-3599 } \\
\text { https://doi.org/10.1109/ A SC.2009.2018459 }\end{array}$ \\
\hline Issue Date & $2009-06$ \\
\hline Doc URL & http:/hdl .handle.net/2115/38987 \\
\hline Rights & $\begin{array}{l}\text { @2009 IEEE. Personal use of this material is permitted. However, permission to reprint/republish this material for } \\
\text { advertising or promotional purposes or for creating new collective works for resale or redistribution to servers or lists, } \\
\text { or to reuse any copyrighted component of this work in other works must be obtained from the IEEE. }\end{array}$ \\
\hline Type & article \\
\hline File Information & 19-3_p3595-3599.pdf \\
\hline
\end{tabular}

Instructions for use 


\title{
Precise Measurement of IMD Behavior in 5-GHz HTS Resonators and Evaluation of Nonlinear Microwave Characteristics
}

\author{
Shunichi Futatsumori, Member, IEEE, Masato Furuno, Takashi Hikage, Member, IEEE, \\ Toshio Nojima, Member, IEEE, Akihiko Akasegawa, Teru Nakanishi, and Kazunori Yamanaka
}

\begin{abstract}
The intermodulation distortion (IMD) of a 5-GHz HTS resonator is precisely measured and a nonlinear analysis is conducted. When designing a radio communication system that uses an HTS microwave device, the device's IMD characteristic is one of the most important problems that must be quantitatively evaluated. The amplitudes of third-order IMD (IMD3) and higher-order IMD, which are generated in an HTS resonator given a two-tone fundamental signal, are measured in detail using a fundamental signal cancellation circuit. Moreover, the relative phase of IMD3 is obtained by using a novel measurement system constructed around a reference IMD3 generator. The measured IMD3 phase shows a drastic change in a relatively low IMD3 amplitude region. In addition, the measured resonator exhibits strong higher-order IMD. A nonlinear evaluation using complex power series representation confirms that the drastic phase change may be due to the higher-order distortions present in IMD3.
\end{abstract}

Index Terms-High-temperature superconductors, intermodulation distortion, nonlinear response, superconducting microwave devices.

\section{INTRODUCTION}

$\mathbf{P}$ LANAR microwave filters that employ thin-film hightemperature superconducting (HTS) materials offer, simultaneously, steep skirt characteristics, low insertion loss, and compact implementations. However, the nonlinear responses of these devices limit their practical applications in wireless communication devices [1], [2]. These limitations are usually observed in high-power transmitting filters or sometimes in low-power receiving filters. The generation of intermodulation distortion (IMD) is one of the most important problems with regard to the nonlinear characteristics. This is because laws and technical specifications set strict limits of IMD generation to avoid co-channel interference or adjacent-channel interference [3]. Different origins of the nonlinear responses, which depend

Manuscript received August 25, 2008. First published May 26, 2009; current version published July 15, 2009. This work was partly supported by "Research and development of fundamental technologies for advanced radio frequency spectrum sharing in mobile communication systems" from the Ministry of Internal Affairs and Communications (MIC) of Japan.

S. Futatsumori, M. Furuno, T. Hikage, and T. Nojima are with the Graduate School of Information Science and Technology, Hokkaido University, Sapporo, Hokkaido 060-0814, Japan (e-mail: futatsumori@emwtinfo.ice.eng.hokudai.ac.jp; furuno@wtemc.ist.hokudai.ac.jp; hikage@wtemc. ist.hokudai.ac.jp; nojima@wtemc.ist.hokudai.ac.jp).

A. Akasegawa, T. Nakanishi, and K. Yamanaka are with Fujitsu LTD \& Fujitsu Laboratories LTD., Atsugi, Kanagawa 243-0197, Japan (e-mail: akasegawa.akihi@jp.fujitsu.com; t-naka@jp.fujitsu.com; yamanaka.kaz-02@jp.fujitsu.com).

Digital Object Identifier 10.1109/TASC.2009.2018459 on the RF field applied in HTS microwave devices, have been reported and the debate still continues [4]. Intrinsic effects such as nonlinear Meissner effects or pair-breaking current can play an important role in the generation of nonlinear characteristics [5]. In addition, the nonlinearities can also be caused by extrinsic effects such as superconductor weak links or Josephson junctions at grain boundaries [6].

In an effort to investigate the origin of the nonlinear response, the phase of third harmonic distortion in HTS microwave devices was measured by Booth et al. [7], [8]. They evaluated the phase using a large-signal vector network analyser and clarified that nonlinear inductances, which are caused by pair-breaking effects, are dominant physical factors behind the nonlinear response of the device measured $\left(\mathrm{YBa}_{2} \mathrm{Cu}_{3} \mathrm{O}_{7-\delta}\right.$ coplanar waveguide transmission line at $76 \mathrm{~K}$ ). We have developed high-power transmitting HTS filters in the GHz region that are aimed at mobile base stations [9], [10]. Given this background, the purpose of this paper is to clarify and to evaluate the precise IMD behavior of HTS microwave devices from the application standpoint.

In this paper, the third-order IMD (IMD3) amplitude and phase characteristics as a function of input power are experimentally determined in detail. First, we measure the amplitude of IMD3 and higher-order IMDs generated in a 5-GHz HTS resonator fabricated as a YBCO thin-film; wide dynamic range is achieved through the use of a fundamental signal cancellation circuit. Second, the relative phase of IMD3 is measured using a two-tone fundamental signal. The constructed measurement system is based on a vector network analyser and a reference IMD3 generator. Finally, the relationship between IMD amplitudes and phases are evaluated by applying a complex power series representation. The shift in IMD3 phase is elucidated from the measured amplitude of IMD3 and higher-order IMDs.

\section{MEASUREMENT OF IMD AMPLITUdE AND PHASE}

\section{A. 5-GHz HTS Resonator for Measurements}

The HTS microwave device examined here is a 5-GHz openring resonator, with bandstop characteristic, fabricated using a double-sided $0.5 \mu \mathrm{m}$ YBCO thin-film deposited on a $0.5 \mathrm{~mm}$ thick $\mathrm{MgO}$ substrate [11]. Figs. 1(a) and 1(b) show the structure of the resonator and its measured $S$-parameter response at $50 \mathrm{~K}$, respectively. The total length of the resonator is adjusted to equal one half the wavelength of the resonance frequency. 


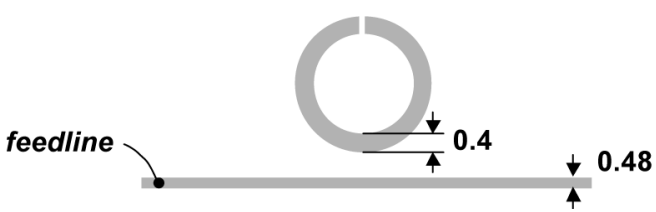

(a)

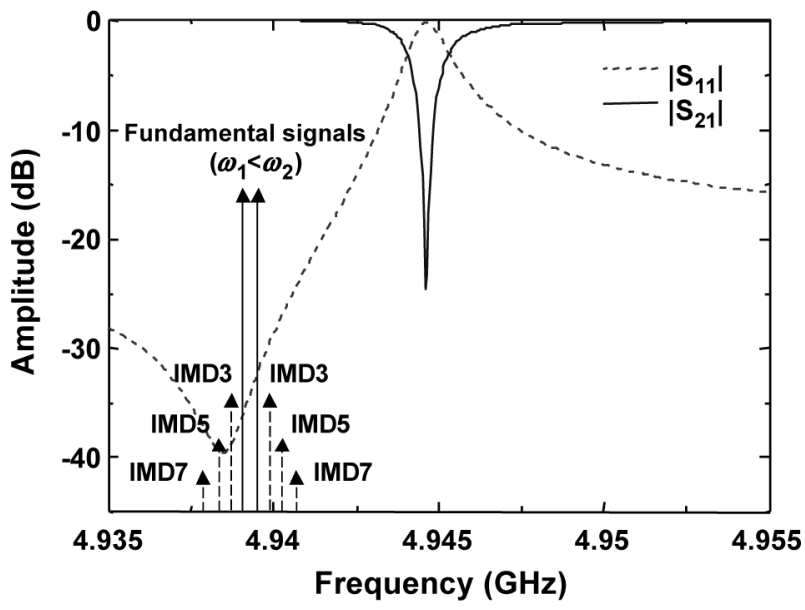

(b)

Fig. 1. The 5-GHz HTS open-ring resonator used in measurements. (a) Structure of the resonator. (all dimensions in millimeters) (b) The measured $S$-parameter response of the resonator at $50 \mathrm{~K}$. The dashed and solid lines represent $\left|S_{11}\right|$ and $\left|S_{21}\right|$ response, respectively. In addition, the condition of IMD measurements is shown. The two-tone fundamental signal is input in the passband region.

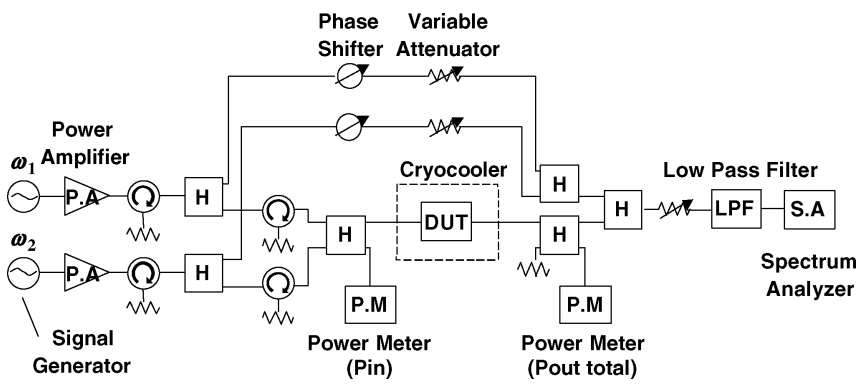

Fig. 2. Schematic diagram of the experimental set-up for IMD measurements using two-tone signal and a 5-GHz HTS resonator, denoted by DUT.

The resonator substrate is mechanically packaged in an aluminum container. The unloaded quality factor of the resonator is 75,100 at $50 \mathrm{~K}$.

\section{B. IMD3 and Higher-Order IMDs Amplitude Measurements}

The amplitudes of IMD3 and higher-order IMDs such as fifthorder (IMD5) and seventh-order IMD (IMD7) are measured to obtain, in detail, the nonlinear characteristics of the 5-GHz HTS resonator. As shown in Fig. 2, two-tone IMD measurement is carried out with a fundamental signal vector cancellation circuit [12]. This experimental set-up is employed for IMD and harmonics measurements of HTS devices [13]. Since the fundamental signal can be suppressed more than $60 \mathrm{~dB}$, this arrangement enables to measure the IMDs without being affected by nonlinear effects of the instruments. This system allows us to measure the IMDs with a wide dynamic range and high-accuracy.

The IMD measurements using a bandstop resonator have been reported in [14]. In the reference, the fundamental signals

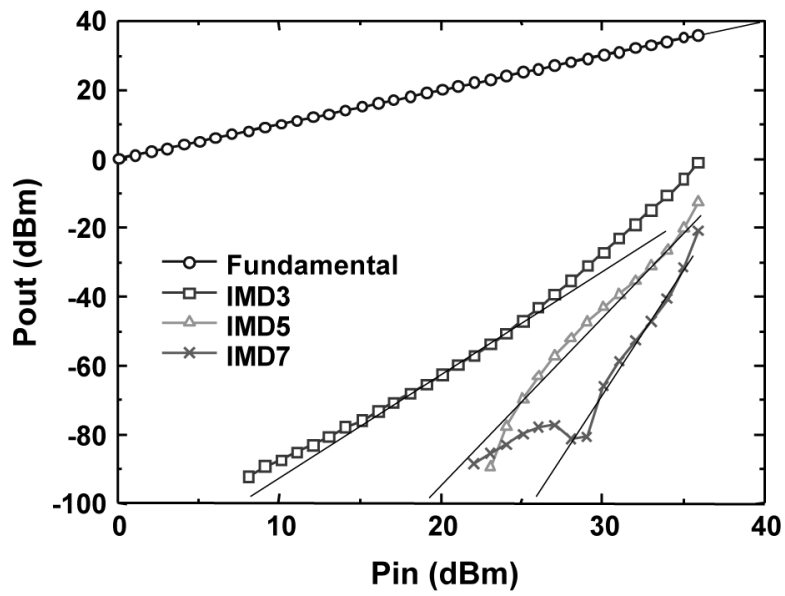

Fig. 3. Measured IMD values of the resonator at $50 \mathrm{~K}$. The open circles represent the amplitude of fundamental signal. In addition, the open boxes, open triangles, and stars represent the amplitudes of IMD3 $\left(2 \omega_{2}-\omega_{1}\right)$, IMD5 $\left(3 \omega_{2}-\right.$ $\left.2 \omega_{1}\right)$, and IMD7 $\left(4 \omega_{2}-3 \omega_{1}\right)$, respectively. (The solid straight lines are the fitting results for the fundamental signal, IMD3, IMD5, and IMD7 with slopes of $1,3,5$, and 7 , respectively.)

are inputted in the stopband frequency region. This configuration has the advantage that the fundamental signal can be cancelled by bandstop characteristics. On the other hand, we have been developing HTS bandstop transmitting filters as another project [9], [11]. This is the reason why a bandstop resonator is used for the measurement. Assuming that the transmitting signals pass through the filter, fundamental signals are inputted in the passband region. Fig. 3 shows an example of the measured IMDs at $50 \mathrm{~K}$. In this case, the fundamental signals are input at $5 \mathrm{MHz}$ and $5.5 \mathrm{MHz}$ offset from below the resonance frequency. These frequencies are in the passband and match the condition shown in Fig. 1.

By using the fundamental signal cancellation circuit, the IMDs could be measured in detail at low power levels. Our measurements confirmed that these IMDs exist even in the very low input power region. In addition, relatively high level IMD5 and IMD7 are observed in the resonator. The measured value of IMD3 is $-41.0 \mathrm{dBc}$ at the fundamental signal of $35.0 \mathrm{dBm}$. In addition, IMD5 and IMD7 are $-55.3 \mathrm{dBc}$ and $-66.4 \mathrm{dBc}$ at the same fundamental signal power. This means that the measured resonator has nonlinear response with strong higher-order nonlinearity. The higher-order distortion included in IMD3 cannot be ignored in these IMD regions.

\section{IMD3 Relative Phase Measurements}

IMD3 phase is obtained using a measurement system based on a reference IMD3 generator. IMD3 phase is important in characterizing the responses of nonlinear devices [15]. The third-order nonlinear distortion phase can be calculated from the measured result of AM-AM and AM-PM conversion of a single-tone fundamental signal [16]. However, this is less accurate when strong higher-order distortions are included in the fundamental signal. To measure IMD3 phase directly, a complicated and accurate measurement system is required. Some measurement techniques that can directly measure IMD3 phase have been reported [17]-[19]. Our measurement configuration is a newly developed configuration based on these techniques. 


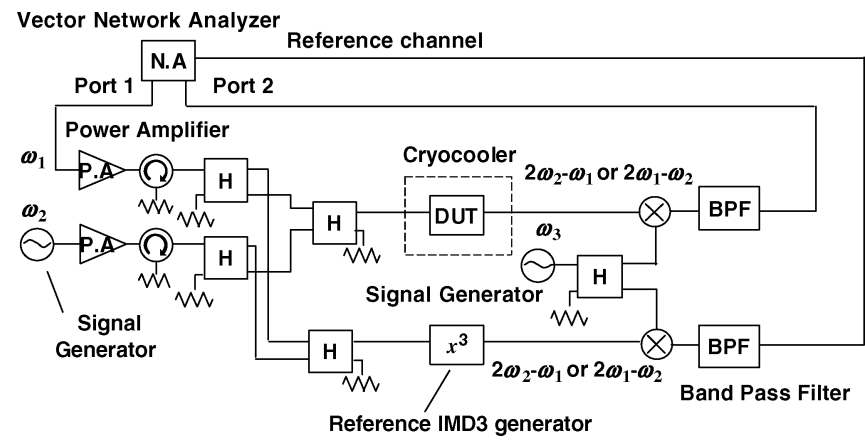

Fig. 4. Schematic diagram of the experimental set-up for IMD3 relative phase measurements using two-tone signal and a 5-GHz HTS resonator. $x^{3}$ represent reference IMD3 generator.

Fig. 4 shows the schematic diagram of the experimental set-up for IMD3 relative phase measurements using a two-tone fundamental signal. This set-up mainly consists of a reference IMD3 generator, a vector network analyser, signal generators, frequency mixers, and bandpass filters. The phase of IMD3 produced by the resonator is compared against that of the generator. The input power of the IMD3 generator is kept constant. This means that the amplitude and phase of IMD3 in the generator has a constant value. In addition, to select only IMD3, $5 \mathrm{GHz}$ signals are downconverted to $10 \mathrm{MHz}$ band. The narrow bandpass filter with $3 \mathrm{~dB}$ bandwidth of $1.8 \mathrm{kHz}$ suppresses the unwanted signals by more than $60 \mathrm{~dB}$. The vector network analyser is operated in frequency offset mode and uses the IMD3 of the generator as a reference channel signal.

Examples of the measured relative phase of the fundamental signal $\left(\omega_{2}, \omega_{1}<\omega_{2}\right)$ and IMD3 $\left(2 \omega_{2}-\omega_{1}\right)$ at $50 \mathrm{~K}$ and $65 \mathrm{~K}$ are shown in Figs. 5 and 6, respectively. In this case, the fundamental signals are input at $4 \mathrm{MHz}$ and $7 \mathrm{MHz}$ offset from below the resonance frequency. The values on the plots show the phase change from that of the minimum input power tested here, 21.8 $\mathrm{dBm}$.

Note that the absolute phase of the fundamental signal and IMD3 are not the same at the minimum input power, since the relative phases are shown. Figs. 5 and 6 also show the amplitudes of IMD3 $\left(2 \omega_{2}-\omega_{1}\right)$ and IMD5 $\left(3 \omega_{2}-2 \omega_{1}\right)$. The IMD3 relative phase obtained in this measurement condition is drastically changed in the region where the amplitude of IMD3 is still low. As shown in Fig. 5, when the amplitude of IMD3 is about $-40 \mathrm{dBc}$ of the fundamental signal, the relative phase of IMD3 changes by more than -34 degrees at $50 \mathrm{~K}$.

\section{Evaluation of Nonlinear Characteristics}

Circuit-based models which enable prediction of the nonlinearity of HTS microwave devices have already been proposed and their validity confirmed [4], [20]. In particular, characteristics of the IMD3 such as temperature and power dependences are sufficiently estimated by using the models. However, the IMD characteristics reported in the pervious section are measured at relatively high input powers (between $20 \mathrm{dBm}$ to 40 $\mathrm{dBm}$ ). In addition, the measured IMD characteristics show high-level higher-order IMDs. The IMD3 may be affected by the higher-order distortions in these IMD regions. As a result, the nonlinear analysis including higher-order distortions is required.

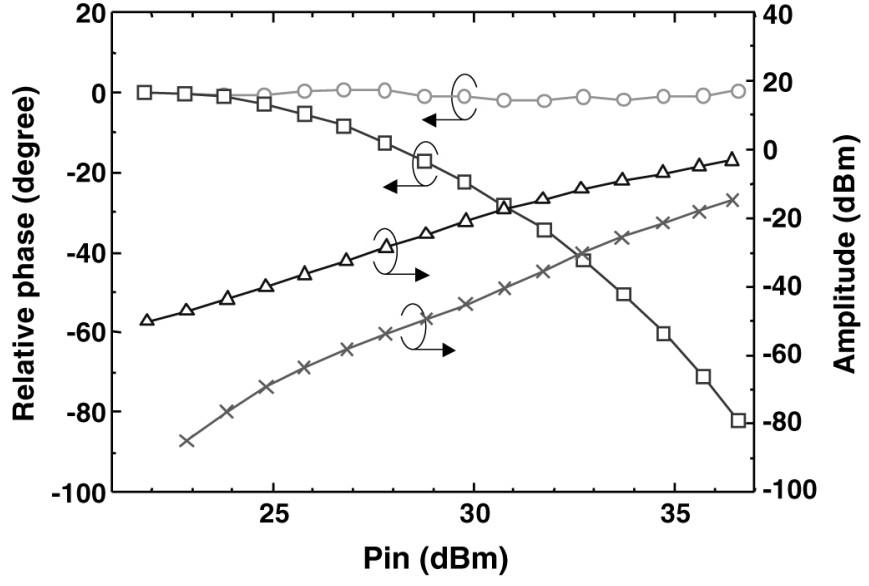

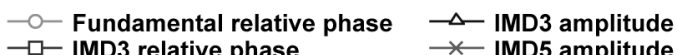

Fig. 5. Measured relative phase of the fundamental signal $\left(\omega_{2}, \omega_{1}<\omega_{2}\right)$ and IMD3 $\left(2 \omega_{2}-\omega_{1}\right)$ of the resonator at $50 \mathrm{~K}$. The open circles and open boxes represent the relative phase of fundamental signal and IMD3, respectively. In addition, the amplitude of IMD3 $\left(2 \omega_{2}-\omega_{1}\right)$ and IMD5 $\left(3 \omega_{2}-2 \omega_{1}\right)$ are obtained by using IMD measurement system shown in Fig. 2. The open triangles and stars represent the amplitude of IMD3 and IMD5, respectively.
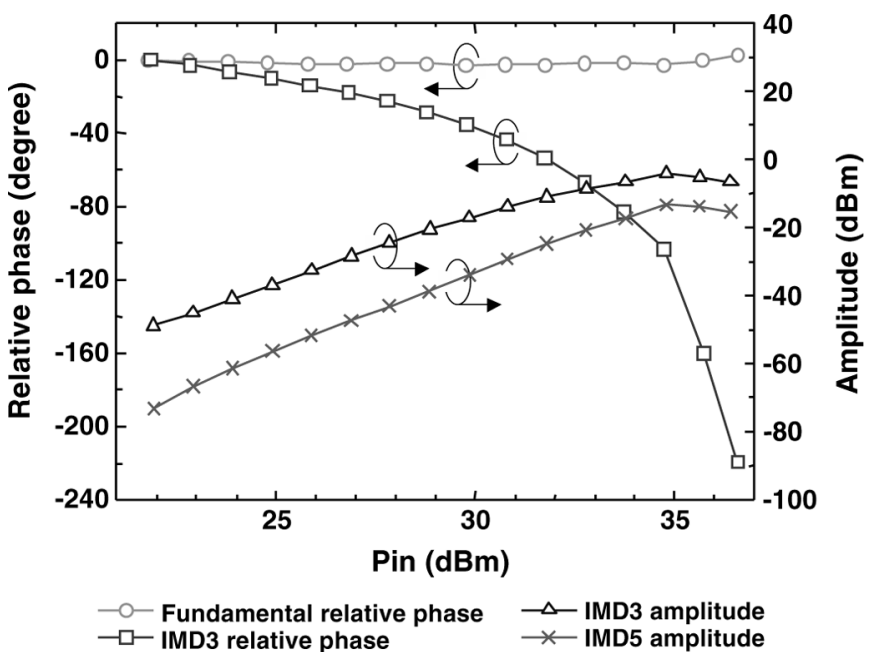

Fig. 6. Measured relative phase of the fundamental signal $\left(\omega_{2}, \omega_{1}<\omega_{2}\right)$ and IMD3 $\left(2 \omega_{2}-\omega_{1}\right)$ of the resonator at $65 \mathrm{~K}$. In addition, the amplitude of IMD3 $\left(2 \omega_{2}-\omega_{1}\right)$ and IMD5 $\left(3 \omega_{2}-2 \omega_{1}\right)$ are shown.

The measured nonlinear characteristics are evaluated using a nonlinear model that assumed memoryless AM-AM and AM-PM conversion. The input-output voltage transfer function of the device is approximated by a complex power series, which is equivalent to a quadrature nonlinear model [16], [21]. Complex power series have an advantage in that they can express each order distortions based on nonlinear transfer coefficients. The transfer coefficients are complex numbers and consist of amplitude and phase coefficients. This representation is useful when the higher-order distortions and the phase of each order distortion are taken into account. By using this representation, the relationship between higher-order distortions and phase change in IMDs can be investigated. Only odd-order terms are employed for the modeling, since fundamental signals and IMDs, which are generated in near fundamental signals, are analysed in this section. 


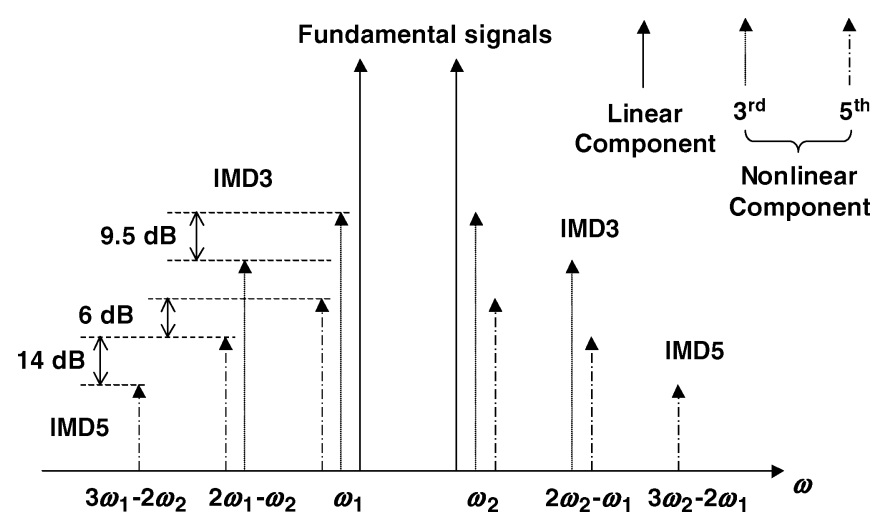

Fig. 7. Output spectrum of the nonlinear model in response to a two-tone fundamental signal represented by fifth-order complex power series. The contributions of each order nonlinear component to fundamental signals, IMD3, and IMD5 are described.

The following general expression described in [16] allows both amplitude and phase nonlinearities to be handled simultaneously:

$$
v_{o}=A_{1} v_{i}+\operatorname{Re}\left[\sum_{n=2}^{5} \boldsymbol{A}_{\boldsymbol{n}}\left\{v_{i}^{n}+j H\left(v_{i}^{n}\right)\right\}\right]
$$

where $v_{i}$ and $v_{o}$ are input and output voltage, respectively, and $A_{\boldsymbol{n}}=A_{n} e^{j \varphi_{n}}(\mathrm{n}=2,3,4,5)$ are the nonlinear transfer coefficients expressed by complex numbers. This model adopts fifth-order nonlinearity because the relationship between IMD3 and IMD5 is discussed. In addition, $A_{n}$ and $\varphi_{n}$ are amplitude and phase coefficients, respectively. $A_{1}$ is the linear coefficient. $H\left(v_{i}^{n}\right)$ are the Hilbert transforms of $v_{i}^{n}$.

Assuming that a two-tone fundamental signal with same amplitude is input to the device:

$$
v_{i}=\frac{1}{2} a\left(\cos \omega_{1} t+\cos \omega_{2} t\right)
$$

where $a$ is input voltage amplitude. The output IMD3 lower sideband (LSB) component, $v_{\text {IMD3L }}$, and the IMD5 LSB component, $v_{\mathrm{IMD} 5 \mathrm{~L}}$, are expressed as

$$
\begin{aligned}
v_{\text {IMD3L }} & =\operatorname{Re}\left[\left(\frac{1}{32} a^{3} \boldsymbol{A}_{\mathbf{3}}+\frac{25}{256} a^{5} \boldsymbol{A}_{\mathbf{5}}\right) \cdot e^{j\left(2 \omega_{1}-\omega_{2}\right) t}\right] \\
& =\operatorname{Re}\left[\left(\boldsymbol{D}_{\mathbf{3}}+5 \boldsymbol{D}_{\mathbf{5}}\right) \cdot e^{j\left(2 \omega_{1}-\omega_{2}\right) t}\right] \\
v_{\text {IMD5L }} & =\operatorname{Re}\left[\frac{5}{256} a^{5} \boldsymbol{A}_{\mathbf{5}} \cdot e^{j\left(3 \omega_{1}-2 \omega_{2}\right) t}\right] \\
& =\operatorname{Re}\left[\boldsymbol{D}_{\mathbf{5}} \cdot e^{j\left(3 \omega_{1}-2 \omega_{2}\right) t}\right]
\end{aligned}
$$

where $\boldsymbol{D}_{3}$ and $\boldsymbol{D}_{5}$ are third and fifth-order distortion components, respectively. The upper sideband components are also obtained in a similar way.

Fig. 7 shows the output spectrum of the nonlinear model in response to a two-tone fundamental signal. This figure indicates that IMD3 is the sum of third and fifth-order components. In addition, Figs. 8(a) and 8(b) show the vector representation of IMD3 LSB component, $v_{\mathrm{IMDL} 3}$, expressed in (3). They plot the resultant vectors of IMD3, where ratios of third and fifth-order distortions are different. When the fifth-order component $5 \boldsymbol{D}_{5}$ has relatively low amplitude level compared to the third-order

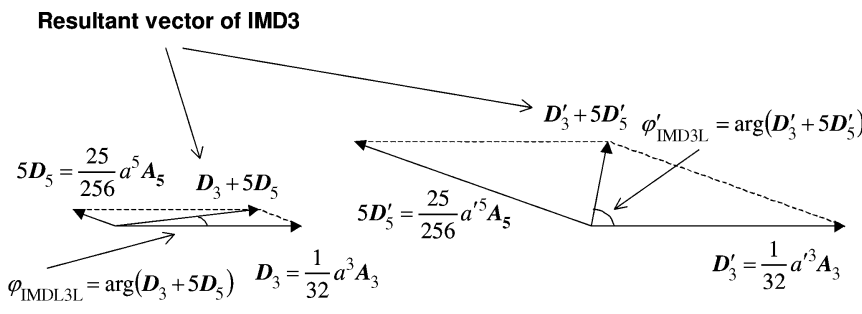

(a)

(b)

Fig. 8. Vector representation of IMD3 LSB component, $v_{\mathrm{IMDL} 3}$, expressed in (3). $\varphi_{\text {IMD3L }}$ represent the phase of IMD3 LSB. (a) Resultant vector of IMD3, $\boldsymbol{D}_{3}+5 \boldsymbol{D}_{5}$, where fifth-order component $\boldsymbol{D}_{5}$ has relatively low amplitude level compared to third-order component $\boldsymbol{D}_{3}$. (b) Resultant vector of IMD3, $\boldsymbol{D}_{3}^{\prime}+$ $5 \boldsymbol{D}_{5}^{\prime}$, where fifth-order component $\boldsymbol{D}_{5}^{\prime}$ has almost the same amplitude level as third-order component $\boldsymbol{D}_{3}^{\prime}$.

component $\boldsymbol{D}_{3}$, the phase of IMD3, $\varphi_{\mathrm{IMD} 3 \mathrm{~L}}$, is slightly shifted from third-order component $\boldsymbol{D}_{3}$. However, when $5 \boldsymbol{D}_{5}^{\prime}$ has almost the same amplitude level as $\boldsymbol{D}_{3}^{\prime}, \varphi_{\text {IMD3L }}^{\prime}$ is drastically shifted form $\boldsymbol{D}_{3}^{\prime}$. Note that $A_{n}$ and $\varphi_{n}$ are not changed depending on the input voltage amplitude.

A comparison of the measured amplitudes of IMDs in Fig. 5 shows that the difference in IMD3 and IMD5 is less than $12 \mathrm{~dB}$ at the input power of $36.4 \mathrm{dBm}$. At this input power, the relative phase of IMD3 changes -82.0 degrees. In the nonlinear model representation, the fifth-order distortion included in IMD3 is approximately $14 \mathrm{~dB}$ higher than IMD5. This relationship is directly calculated from the coefficient of $D_{5}$ in (3) and (4). This means that the amplitude level of the fifth-order distortion included in IMD3 is near to or larger than that of third-order distortion. The phase change in IMD3 is caused by the high level fifth-order distortion. In addition, IMD5 amplitudes at 65 $\mathrm{K}$ (shown in Fig. 6) are higher than those at $50 \mathrm{~K}$ (shown in Fig. 5), for the same input powers. This explains why large shifts in IMD3 phases are observed at $65 \mathrm{~K}$, compared with those at $50 \mathrm{~K}$.

Since actual devices exhibit more higher-order distortions as shown in Fig. 3, this approximation, based on the fifth-order complex power series representation, is rather simple. However, the analysis confirms that the dominant reason behind the IMD3 phase changes in these IMD regions is the presence of strong higher-order nonlinearities.

\section{CONCLUSIONS}

The nonlinear characteristics of 5-GHz HTS resonators were measured in detail. Amplitude and phase measurements of IMDs demonstrated strong higher-order nonlinearities of the measured device in a relatively low IMD3 amplitude region (where IMD3 is less than $-60 \mathrm{dBc}$ below the fundamental signal). In addition, based on the measured results and a nonlinear model, we clarified that the phase change in IMD3 is mainly due to strong higher-order distortions.

Note that the physical origin of the strong higher-order distortions was not discussed and further investigation is needed. We plan to carry out a more detailed analysis of the IMD characteristics noted herein, which will include the existence of the memory effect, and additional quantitative evaluations of the IMD amplitude and phase. 


\section{REFERENCES}

[1] Z.-Y. Shen et al., "Power handling capability improvement of hightemperature superconducting microwave circuits," IEEE Trans. Appl. Supercond., vol. 7, no. 2, pp. 2446-2453, Jun. 1997.

[2] S. Sato, J. Kurian, and M. Naito, "Third-order intermodulation measurements of microstrip bandpass filter based on high-temperature superconductors," IEEE Trans. Microwave Theory Tech., vol. 52, no. 12, pp. 2658-2663, Dec. 2004.

[3] For example, 3GPP TS-25.141 V6.8.0 (2004-12), Third Generation Partnership Project, 2004.

[4] D. E. Oates, S.-H. Park, D. Agassi, G. Koren, and K. Irgmaier, "Temperature dependence of intermodulation distortion in YBCO: Understanding nonlinearity," IEEE Trans. Appl. Supercond., vol. 15, no. 2, pp. 3589-3595, Jun. 2005.

[5] D. Xu, S. K. Yip, and J. A. Sauls, "Nonlinear Meissner effect in unconventional superconductors," Phys. Rev. B, vol. 51, no. 22, pp. 16233-16253, Jun. 1995.

[6] A. T. Findikoglu et al., "Power-dependent microwave properties of superconducting $\mathrm{YBa}_{2} \mathrm{Cu}_{3} \mathrm{O}_{7-\mathrm{x}}$ films on buffered polycrystalline substrates," Appl. Phys Lett., vol. 70, no. 24, pp. 3293-3295, Jun. 1997.

[7] J. C. Booth, K. Leong, S. A. Schima, J. A. Jargon, and D. C. DeGroot, "Design and characterization of a superconducting nonlinear reference device," in 62nd ARFTG Conf., Dig. Fall 2003, Dec. 2003, pp. 61-70.

[8] J. C. Booth, "Phase-sensitive measurement of nonlinearity in high-temperature superconductor thin films," IEEE Trans. Appl. Supercond., vol. 15, no. 2, pp. 1000-1003, Jun. 2005.

[9] S. Futatsumori, "A 5 GHz high-temperature superconducting reactiontype transmitting filter based upon split open-ring resonators," Supercond. Sci. Technol., vol. 21, no. 4, pp. 1-8, Apr. 2008.

[10] K. Yamanaka, A. Akasegawa, M. Kai, and T. Nakanishi, "RF power dependence of microstrip disk resonators with YBCO films for $4 \mathrm{GHz}$ band," IEEE Trans. Appl. Supercond., vol. 15, no. 2, pp. 1024-1027, Jun. 2005.
[11] S. Futatsumori, "HTS split open-ring resonators with improved power handling capability for reaction-type transmitting filters," IET Electron. Lett., vol. 43, no. 17, pp. 956-957, Aug. 2007.

[12] P. Blount, M. Olson, R. Tshudy, D. Foote, and C. Trantanella, "An automated phase cancellation method for measuring ultra-high third order intercept points," in IEEE MTT-S Int. Microwave Symp. Dig., Jun. 2004, pp. 1727-1730.

[13] J. Mateu, J. C. Booth, and S. A. Schima, "Frequency tuning and spurious signal generation at microwave frequencies in ferroelectric SrTiO3 thin-film transmission lines," IEEE Trans. Microwave Theory Tech., vol. 55, no. 2, pp. 391-396, Feb. 2007.

[14] B. A. Willemsen and B. H. King, "Microwave intermodulation in high-Tc superconducting microstrip resonators," IEEE Trans. Appl. Supercond., vol. 9, no. 2, pp. 4181-4184, June 1999.

[15] J. H. K. Vuolevi, T. Rahkonen, and P. A. Manninen, "Measuremen technique for characterizing memory effects in RF power amplifiers," IEEE Trans. Microwave Theory Tech., vol. 49, no. 8, pp. 1383-1389, Aug. 2001.

[16] T. Nojima and T. Konno, "Cuber predistortion linearizer for relay equipment in $800 \mathrm{MHz}$ in band land mobile telephone system," IEEE Trans. Veh. Technol., vol. 34, no. 4, pp. 169-177, Nov. 1985.

[17] N. Suematsu, Y. Iyama, and O. Ishida, "Transfer characteristics of $\mathrm{IM}_{3}$ relative phase for a GaAs FET amplifier," IEEE Trans. Microwave Theory Tech., vol. 45, no. 12, pp. 2509-2514, Dec. 1997.

[18] Y. Yang, J. Yi, J. Nam, B. Kim, and M. Park, "Measurement of twotone characteristics of high-power amplifiers," IEEE Trans. Microwave Theory Tech., vol. 49, no. 3, pp. 568-571, Mar. 2001.

[19] S.-Y. Lee, Y.-S. Lee, and Y.-H. Jeong, "A novel phase measurement technique for IM3 components in RF power amplifiers," IEEE Trans. Microwave Theory Tech., vol. 54, no. 1, pp. 451-457, Jan. 2006.

[20] C. Collado, J. Mateu, and M. O'Callaghan, "Analysis and simulation of the effects of distributed nonlinearities in microwave superconducting devices," IEEE Trans. Appl. Supercond., vol. 15, no. 1, pp. 26-39, Mar. 2005.

[21] A. R. Kaye, D. A. George, and M. J. Eric, "Analysis and compensation of bandpass nonlinearities for communications," IEEE Trans. Commun., vol. 20, no. 5, pp. 965-972, Oct. 1972. 